\title{
Performance Analysis of Hybrid Estimation Algorithms
}

\author{
Inseok Hwang, Hamsa Balakrishnan, and Claire Tomlin \\ Hybrid Systems Laboratory \\ Department of Aeronautics and Astronautics \\ Stanford University, Stanford, CA 94305 \\ ishwang, hamsa, tomlinestanford.edu
}

\begin{abstract}
In this paper, we analyze the performance of estimation algorithms for discrete-time stochastic linear hybrid systems. The problem of being able to estimate both the discrete and continuous states of a hybrid system given only the continuous output sequence is a difficult one, and while algorithms [1], [2] exist for this purpose, little has been proved on the limitations of these algorithms, or even the dependence of their performance on system parameters. We find necessary conditions to guarantee the convergence of these hybrid estimation algorithms. We also derive expressions to determine bounds on the discrete mode detection delay. These conditions also provide a method to predict a priori which transitions in a hybrid system are relatively easy to detect, as a function of the system parameters. Finally, we validate our conditions and predictions using first a simple yet illustrative 1-D example, and then a more complex aircraft tracking example.
\end{abstract}

\section{INTRODUCTION}

Many complex systems can be modeled by hybrid systems with a number of discrete modes having different continuous dynamics and discrete transition relations between the modes. The objective of hybrid estimation is to estimate both the mode and the continuous state of a hybrid system at any given time. Hybrid estimators usually consist of the combination of a bank of continuous state estimators (usually Kalman filters), designed for the different discrete modes, and a hypothesis testing (mode selecting) algorithm. The class of hybrid estimators analyzed in this paper addresses a very challenging problem - that of mode detection and state estimation given only the continuous system output data. For hypothesis testing, it is then necessary to use the differences in statistical properties (such as mean and covariance) of the outputs from the different Kalman filters to choose the most likely mode.

Hybrid estimation algorithms have been developed for discrete-time stochastic linear hybrid systems in which the discrete transitions are governed by finite-state Markov chains. The Multiple Model Adaptive Estimation Algorithm (MMAE) [1] is an algorithm in which, for hypothesis testing, the Kalman filter residuals are used to form likelihood functions for the different modes, which are then used as adaptive weights to find the most probable mode. A refinement of the MMAE, the Interacting Multiple Model Algorithm (IMM) [2], mixes the initial conditions of each of the Kalman filters at each time-step. The rationale behind this refinement was to enable keeping information from $N^{2}$ discrete time histories (where $N$ is the number of discrete modes) with just $N$ Kalman filters. [1], [2], [3], [4] give various other hybrid estimation algorithms and their applications.

While several researchers ([4], [5], [6], [7], [8], [9], [10], [11]) have analyzed hybrid estimators for special classes of systems, general analysis techniques for the performance of hybrid estimation algorithms have not been investigated in detail until now. Maybeck [1] gives qualitative reasons for the performance of hybrid estimators and adds that no rigorous general proofs are available for the (asymptotic convergence) properties of the hypothesis conditional probabilities. In this paper, we attempt to remedy this situation and successfully derive conditions under which the hybrid estimates converge exponentially to the exact hybrid states. We determine necessary conditions for mode detection, sufficient conditions for instantaneous mode detection, and bounds on the mode detection delay. The results of our analysis also provide insight into determining which mode transitions are more detectable (faster convergence to true mode) than others and also improving the performance of hybrid estimators. We compare the performance of the MMAE algorithm with that of the IMM algorithm which has been widely (and successfully) used in the area of multiple target tracking. We show analytically why the IMM algorithm has better performance than the MMAE. For brevity, we omit proofs - they are available upon request.

\section{DISCRETE-TIME STOCHASTIC LINEAR HYBRID SYSTEMS} [12]

We consider a discrete-time stochastic linear hybrid system

$$
H:\left\{\begin{array}{cl}
x(k+1) & =A_{i} x(k)+B_{i} u(k)+w_{i}(k) \\
z(k) & =C_{i} x(k)+v_{i}(k)
\end{array}\right.
$$

where $k \in \mathbb{N}, x \in \mathbb{R}^{n}, u \in \mathbb{R}^{l}$ and $z \in \mathbb{R}^{p}$ are the continuous state, control input, and output variables respectively. The index $i \in\{1,2, \cdots, N\}$ represents the discrete state whose evolution is governed by the finite state Markov chain

$$
\mu(k+1)=\Pi \mu(k)
$$

where $\Pi=\left\{\pi_{i j}\right\} \in \mathbb{R}^{N \times N}$ is the mode transition matrix and $\mu(k) \in \mathbb{R}^{N}$ is the mode probability at time $k$. The system matrices $A_{i} \in \mathbb{R}^{n \times n}, B_{i} \in \mathbb{R}^{n \times l}$, and $C_{i} \in \mathbb{R}^{p \times n}$ for $i \in\{1,2, \cdots, N\}$ are assumed known. We denote the 
covariance of the initial state $x\left(k_{0}\right)$ as $\pi_{0} \in \mathbb{R}^{N}$, and assume that the process noise $w_{i}(k)$ and the measurement noise $v_{i}(k)$ are uncorrelated, zero-mean white Gaussian sequences with the covariance matrices $E\left[w_{i}(k) w_{i}(k)^{T}\right]=Q_{i} \in \mathbb{R}^{n \times n}$ and $E\left[v_{i}(k) v_{i}(k)^{T}\right]=R_{i} \in \mathbb{R}^{p \times p}$ respectively. $E[\cdot]$ and $(\cdot)^{T}$ denote expectation and matrix transpose respectively. It is assumed that $w_{i}(k)$ and $v_{i}(k)$ are both uncorrelated with the initial state, i.e., $E\left[x\left(k_{0}\right) w_{i}(k)^{T}\right]=E\left[x\left(k_{0}\right) v_{i}(k)^{T}\right]=0$. We define $Z(k)=\{z(0), \cdots, z(k)\}$ as the measurement sequence up to time $k$. Since the state evolution of a hybrid system has continuous trajectories as well as discrete jumps, we define a hybrid time trajectory:

Definition 1: (Hybrid time trajectory) A hybrid time trajectory is a sequence of intervals $\left[k_{0}, k_{1}-1\right]\left[k_{1}, k_{2}-\right.$ $1] \cdots\left[k_{i}, k_{i+1}-1\right] \cdots$ where $k_{i}(i \geq 1)$ is the time at which the $i$-th discrete state transition occurs.

In this paper, by 'exponential convergence of a hybrid estimator' we mean:

Definition 2: (Exponential convergence of a hybrid estimator) Given a hybrid system $H$ with $N$ discrete modes, we say that a hybrid estimator is exponentially convergent if its discrete state estimate exhibits correct identification of the discrete-state transition sequence of the original system after a finite number of steps; and at any given time, the continuous state estimate is unique, with a mean estimation error which is exponentially bounded to zero across transitions.

\section{HYBRID ESTIMATION ALGORITHMS}

In this section, we consider a generic hybrid estimation algorithm [1] for the discrete-time stochastic linear hybrid system (1)-(2). From Bayesian estimation [1], the continuous state estimate is the conditional mean:

$$
\hat{x}(k+1)=E[x(k+1) \mid Z(k+1)]
$$

Let $p(\cdot \mid \cdot)$ be the conditional probability density function, given by:

$$
p(x(k) \mid Z(k))=\sum_{i=1}^{N} \frac{p\left(x(k), Z(k), m_{i}(k)\right)}{p(Z(k))}
$$

where $m_{i}(k)$ denotes the event that the mode at time $k$ is $i$. Thus, the state estimate (3) is

$$
\hat{x}(k+1)=\sum_{i=1}^{N} \hat{x}_{i}(k+1) p\left(m_{i}(k+1) \mid Z(k+1)\right)
$$

where $\hat{x}_{i}(k+1)=\int_{-\infty}^{\infty} x(k+1) p\left(x(k+1) \mid Z(k+1), m_{i}(k+\right.$ 1)) $d x(k+1)$ is the mode-conditioned state estimate of $x(k+$ 1) given $m_{i}(k+1) . \hat{x}_{i}(k+1)$ is computed by the state estimator matched to mode $i$. Therefore, the state estimate (4) is a weighted sum of $N$ mode-conditioned state estimates produced by each Kalman filter with the weight $p\left(m_{i}(k+\right.$ $1) \mid Z(k+1))$. The weight is given by

$$
p\left(m_{i}(k+1) \mid Z(k+1)\right)=\frac{\Lambda_{i}(k+1) p\left(m_{i}(k+1) \mid Z(k)\right)}{p(z(k+1) \mid Z(k))}
$$

where $\Lambda_{i}(k+1):=\mathcal{N}\left(r_{i}(k+1) ; 0, S_{i}(k+1)\right)$ is the likelihood function (i.e., $\left.p\left(z(k+1) \mid m_{i}(k+1), Z(k)\right)\right)$ of mode $i, r_{i}(k+$ $1)=z(k+1)-C_{i} \hat{x}_{i}(k+1)$ is the residual produced by the Kalman filter $i, S_{i}(k+1) \in \mathbb{R}^{p \times p}$ is the corresponding residual covariance, and $\mathcal{N}(a ; b, c)$ is the probability at $a$ of a normal distribution with mean $b$ and covariance $c . p\left(m_{i}(k+\right.$ 1) $\mid Z(k))$ is the mode probability estimate at time $k+1$. If the mode transitions are governed by a finite Markov chain, the mode probability estimate can be expressed by

$$
p\left(m_{i}(k+1) \mid Z(k)\right)=\sum_{l=1}^{N} \pi_{i l} p\left(m_{l}(k) \mid Z(k)\right)
$$

Thus, the weight (mode probability) is

$$
\mu_{i}(k+1):=\frac{\Lambda_{i}(k+1)}{c(k+1)} \sum_{l=1}^{N} \pi_{i l} p\left(m_{l}(k) \mid Z(k)\right)
$$

where $c(k+1)$ is a normalization constant. The mode estimate at time $k$ is chosen to be the mode which has the maximum mode probability at that time. The mode probability depends not only on the finite Markov chain but also on the likelihood produced by each Kalman filter. A probabilistic analysis of the sojourn time based purely on the discrete dynamics of the hybrid system would suggest that the typical sojourn time in any mode is very small. However, the assumption that a finite Markov chain models the discrete dynamics well is not very realistic, since most physical hybrid dynamical systems have longer sojourn times. We therefore need to incorporate knowledge from the continuous dynamics (through the likelihood functions) while computing the mode probability. Thus, the accuracy of the mode probability is affected greatly by the likelihood function. The state estimate (4) is

$$
\begin{array}{ll}
\hat{x}(k+1)=\sum_{i=1}^{N} & \hat{x}_{i}(k+1)\left[\frac{1}{c(k+1)} \Lambda_{i}(k+1)\right. \\
& \left.\sum_{l=1}^{N} \pi_{i l} p\left(m_{l}(k) \mid Z(k)\right)\right]
\end{array}
$$

(6)-(7) is referred to as the Multiple Model Adaptive Estimation (MMAE) algorithm [1]. In the MMAE, all individual Kalman filters run independently at every time step. (7) shows that the state estimate depends on the likelihood function; the performance of the hybrid estimator thus greatly depends on the behavior of the likelihood function.

We now describe the general structure of the IMM algorithm [2]. The IMM has the same structure as the MMAE except that it has the Mixing/Interacting step at the start of the estimation process which adjusts the initial conditions for each Kalman filter at the beginning of every time step, using a weighted sum of the state estimates from the previous time step, as shown in Fig. 1. The optimal hybrid estimator which minimizes the mean-square estimation error has to keep track of all the mode histories up to the current time, and the number of such histories grows exponentially with time, making it impossible to implement in practice. The IMM is a suboptimal algorithm which, at each time step, 


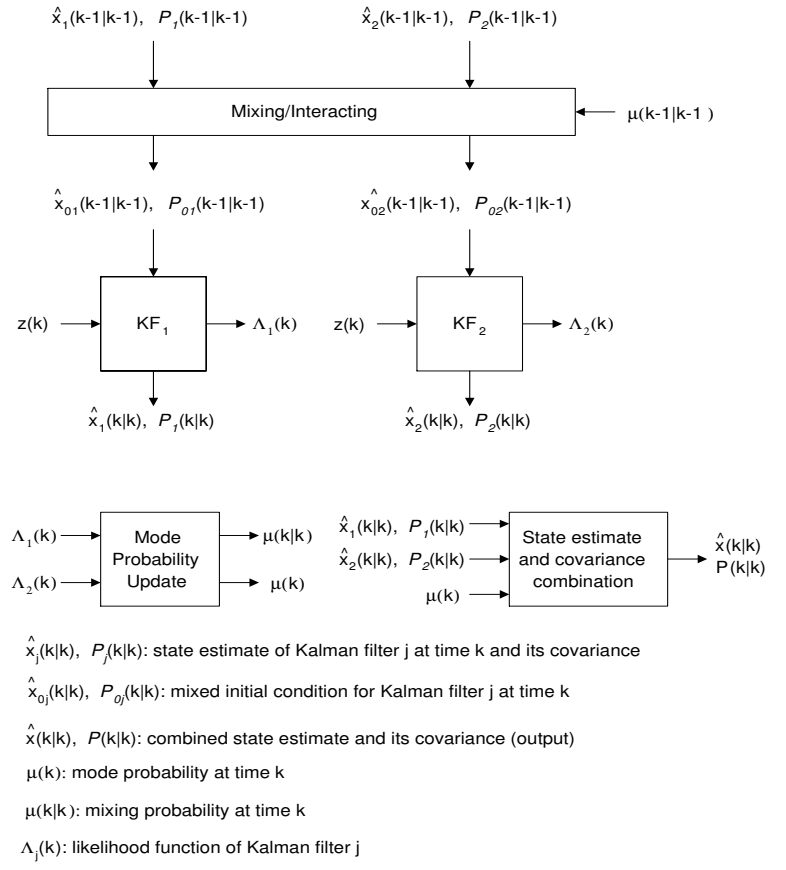

Fig. 1. Structure of the IMM algorithm (two mode) from [13]

keeps information from $N^{2}$ mode histories (where $N$ is the number of modes) with lower complexity, by mixing the $N$ mode histories of the previous time step into inputs to the estimators that produce the $N$ mode histories of the current time step. It thus performs nearly as well as more complex algorithms that keep $N^{2}$ mode histories [2].

\section{Performance analysis}

In this section, we first analyze the performance of the hybrid estimation algorithm (either the MMAE or the IMM) by analyzing the steady-state mean residuals. Since the steadystate analysis gives only necessary conditions on the performance of hybrid estimation, we then analyze the transient behavior of mode probabilities, which are functions of the likelihoods and therefore the residuals.

\section{A. Analysis for the steady-state mean residuals}

Motivated by Caputi [11], we derive the steady-state mean residual for each mode $i$ for the hybrid system (1)-(2). Using the notation in [14], we define:

$$
\begin{aligned}
& \Delta A_{i}:=A_{T}-A_{i} \\
& \Delta B_{i}:=B_{T}-B_{i} \\
& \Delta C_{i}:=C_{T}-C_{i} \\
& \Delta u_{i_{s s}}:=u_{T_{s s}}-u_{i_{s s}}:=\lim _{k \rightarrow \infty} u_{T}(k)-\lim _{k \rightarrow \infty} u_{i}(k) \\
& \hat{x}_{i_{s s}}:=\lim _{k \rightarrow \infty} \hat{x}_{i}(k) \\
& \bar{e}_{i_{s s}}:=\lim _{k \rightarrow \infty} E\left[e_{i}(k)\right]=\lim _{k \rightarrow \infty} E\left[\left(x(k)-\hat{x}_{i}(k)\right)\right]
\end{aligned}
$$

where the subscript $T \in\{1, \cdots, N\}$ represents the true mode. Following a procedure similar to the one adopted by Hanlon et al. [14], we can express the steady-state mean residual for mode $i\left(r_{i_{s s}}\right)$ in terms of the above defined quantities. We assume that $\left[I-\left(I-K_{i} C_{T}\right) A_{T}\right]$ is invertible. If mode $i$ is the correct mode $(i=T)$, then $\bar{r}_{i_{s s}}=0$. If $\bar{r}_{j_{s s}} \neq 0(\forall j \neq i)$, then the correct mode can be detected. However, even if mode $i$ is not the correct mode $(i \neq T)$, the steady-state mean residual for mode $i$ is zero if the following is true: $\left(I-K_{i} C_{T}\right) \Delta A_{i}-K_{i} \Delta C_{i} A_{i}=0 \bigwedge\left(C_{T} \Delta A_{i}+\right.$ $\left.\Delta C_{i} A_{T}-\Delta C_{i} \Delta A_{i}\right)=0 \bigwedge\left(I-K_{i} C_{T}\right) \Delta B_{i}-K_{i} \Delta C_{i} B_{i}=$ $0 \bigwedge\left(C_{T} \Delta B_{i}+\Delta C_{i} B_{T}-\Delta C_{i} \Delta B_{i}\right)=0 \wedge \Delta u_{i_{s s}}=0$. This means that if at least two models are identical and the corresponding control inputs are the same, then the steady-state residuals of both the corresponding modes are zero. In this case, the hybrid estimator will not work. In other words, the performance of the hybrid estimation algorithm depends on the differences between the residuals which in turn arise from model differences and input differences. In the above condition, the first four equalities come from model differences and the last equality comes from input differences. This supports Maybeck's heuristic observation that the performance of the MMAE depends on a significant difference between the residual characteristics [1].

\section{B. Transient analysis for mode probability}

A steady-state Kalman filter is assumed to be used as the state estimator for each mode. For the sake of notational simplicity, we define $\mu_{i}^{-}(k):=\sum_{l=1}^{N} \pi_{i l} \mu_{l}(k-1)$. The condition for correct mode detection at time $k$ is:

$$
\mu_{T}(k)>\mu_{i}(k), \quad \forall i \neq T
$$

Using $\mu_{i}(k):=p\left(m_{i}(k) \mid Z(k)\right)$ in (6), since $\Lambda_{i}(k)=$ $(2 \pi)^{-n / 2}\left|S_{i}\right|^{-1} \exp \left[-\frac{1}{2} \bar{r}_{i}(k)^{T} S_{i}^{-1} \bar{r}_{i}(k)\right]\left(S_{i}=S_{i}^{T}>0\right)$, where $\bar{r}$ is the mean residual, (8) becomes

$$
\begin{aligned}
0 & \leq \bar{r}_{T}(k)^{T} S_{T}^{-1} \bar{r}_{T}(k) \\
& <\bar{r}_{i}(k)^{T} S_{i}^{-1} \bar{r}_{i}(k)+2 \ln \left(\frac{\left|S_{i}\right|}{\left|S_{T}\right|}\right)+2 \ln \left(\frac{\mu_{T}^{-}(k)}{\mu_{i}^{-}(k)}\right)
\end{aligned}
$$

To detect the correct mode exactly for any $k \in \mathbb{N}$, (9) must hold for all $k \in \mathbb{N}(\forall i \neq T)$. If there is a time delay $\left(\delta_{T}\right)$ for correct mode detection when a mode transition into mode $T$ occurs at time $k_{l}\left(l \in \mathbb{N}^{+}\right)$, (9) holds for $k \in\left[k_{l}+\delta_{T}, k_{l+1}\right)$. For the existence of an $\bar{r}_{T}(k)$ satisfying (9), using the properties of the eigenvalues of positive definite matrices [15], we derive the following:

Proposition 1: The correct mode can be detected in $\delta_{T}$ time steps after a mode transition at time $k_{l}$ if there exists $\delta_{T} \in \mathbb{N}^{+}$such that for $k \in\left[k_{l}+\delta_{T}, k_{l+1}\right)\left(l \in \mathbb{N}^{+}, \forall i \neq T\right)$, Condition 1 holds, and either Condition 2 or Condition 3 is true.

$$
\begin{aligned}
& \text { 1) } \bar{r}_{i}(k)^{T} S_{i}^{-1} \bar{r}_{i}(k)+2\left[\ln \left(\frac{\left|S_{i}\right|}{\left|S_{T}\right|}\right)+\ln \left(\frac{\mu_{T}^{-}(k)}{\mu_{i}^{-}(k)}\right)\right]>0 \\
& \text { 2) } \bar{r}_{T}(k)^{T} S_{T}^{-1} \bar{r}_{T}(k)<\bar{r}_{i}(k)^{T} S_{i}^{-1} \bar{r}_{i}(k) \\
& +2\left[\ln \left(\frac{\left|S_{i}\right|}{\left|S_{T}\right|}\right)+\ln \left(\frac{\mu_{T}^{-}(k)}{\mu_{i}^{-}(k)}\right)\right] \\
& \text { 3) }\left\|\bar{r}_{T}(k)\right\|^{2}<\frac{\lambda_{\min }\left(S_{i}^{-1}\right)}{\lambda_{\max }\left(S_{T}^{-1}\right)}\left\|\bar{r}_{i}(k)\right\|^{2} \\
& +\frac{2}{\lambda_{\max }\left(S_{T}^{-1}\right)}\left[\ln \left(\frac{\left|S_{i}\right|}{\left|S_{T}\right|}\right)+\ln \left(\frac{\mu_{T}^{-}(k)}{\mu_{i}^{-}(k)}\right)\right]
\end{aligned}
$$


Condition 3 is a sufficient condition only, and might be a very conservative test for correct mode detection. However, it gives valuable insight into the performance of hybrid estimation. Fast mode detection is dependent not only on the magnitudes of the residuals produced by each Kalman filter but also on the residual covariances. If $\frac{\lambda_{\min }\left(S_{i}^{-1}\right)}{\lambda_{\max }\left(S_{T}^{-1}\right)}$ is small and/or $\frac{\left|S_{i}\right|}{\left|S_{T}\right|}$ is small, it is difficult for Condition 3 to hold and thus to detect the correct mode. Therefore, by checking the eigenvalues and determinant of $S_{i}^{-1}$, we can tell which mode transitions are more detectable than the others. This is similar to the idea of the observability grammian as a measure of which states are more observable than others [16]. If we consider the steady-state residual mean, Condition 3 becomes, $\forall i \neq T$,

$$
\begin{aligned}
\left\|\bar{r}_{T_{s s}}\right\|^{2} & \neq \frac{T}{<} \frac{\lambda_{\min }\left(S_{i}^{-1}\right)}{\lambda_{\max }\left(S_{T}^{-1}\right)}\left\|\bar{r}_{i_{s s}}\right\|^{2} \\
& +\frac{2}{\lambda_{\max }\left(S_{T}^{-1}\right)}\left[\ln \left(\frac{\left|S_{i}\right|}{\left|S_{T}\right|}\right)+\ln \left(\frac{\mu_{T_{s s}^{-}}^{-}}{\mu_{i_{s s}}}\right)\right]
\end{aligned}
$$

Therefore, if the asymptotic behavior of the residuals satisfies (10) and the sojourn times are long enough for the residuals to converge to their steady-state values, then the MMAE is guaranteed to estimate hybrid states correctly.

\section{Mode estimation delay}

In this section, we derive the mode estimation delay $\delta_{i}$ using Condition 3 in Proposition 1. The mean residual of the correct filter at time $k_{l}+\delta_{i}\left(l \in \mathbb{N}^{+}\right)$, when $i=T$, is $\bar{r}_{T}\left(k_{l}+\delta_{T}\right)=C_{T} A_{T}\left[\left(I-K_{T} C_{T}\right) A_{T}\right]^{\delta_{T}-1} \bar{e}_{T}\left(k_{l}\right)$, where $\bar{e}_{T}\left(k_{l}\right)$ is the mean of the estimation error of the correct filter at time $k_{l}$. For the sake of notational simplicity, we define

$$
\begin{aligned}
& F_{T}:=\left(I-K_{T} C_{T}\right) A_{T}, F_{i}:=\left(I-K_{i} C_{T}\right) A_{T} \\
& H_{i}^{x}:=C_{T} \Delta A_{i}+\Delta C_{i} A_{T}-\Delta C_{i} \Delta A_{i} \\
& H_{i}^{u}:=C_{T} \Delta B_{i}+\Delta C_{i} B_{T}-\Delta C_{i} \Delta B_{i} \\
& G_{i}^{x}:=\left(I-K_{i} C_{T}\right) \Delta A_{i}-K_{i} \Delta C_{i} A_{i} \\
& G_{i}^{u}:=\left(I-K_{i} C_{T}\right) \Delta B_{i}-K_{i} \Delta C_{i} B_{i} \\
& L_{i}:=\left[\begin{array}{lll}
C_{T} A_{T} & H_{i}^{x} & H_{i}^{u}
\end{array}\right]
\end{aligned}
$$

At $k_{l}+\delta_{T}$, the correct filter has a (norm) mean residual

$$
\begin{aligned}
\left\|\bar{r}_{T}\left(k_{l}+\delta_{T}\right)\right\| & =\left\|C_{T} A_{T} F_{T}^{\delta_{T}-1} \bar{e}_{T}\left(k_{l}\right)\right\| \\
& \leq \bar{\sigma}\left(C_{T} A_{T}\right) \bar{\sigma}\left(F_{T}\right)^{\delta_{T}-1} \| \bar{e}_{T}\left(k_{l}\right)
\end{aligned}
$$

where $\bar{\sigma}(\cdot)$ denotes the maximum singular value. Similarly, we can show that the norm of the mean residual of the incorrect filter at time $k_{l}+\delta_{T}$ is

$$
\begin{aligned}
\left\|\bar{r}_{i}\left(k_{l}+\delta_{T}\right)\right\| & \geq \underline{\sigma}\left(L_{i}\right)\left\|\bar{e}_{i}\left(k_{l}+\delta_{T}-1\right)\right\| \\
& \geq \underline{\sigma}\left(L_{i}\right) \underline{\sigma}\left(\left[\begin{array}{lll}
F_{i} & G_{i}^{x} & G_{i}^{u}
\end{array}\right]\right)^{\delta_{T}-1}\left\|\bar{e}_{i}\left(k_{l}\right)\right\|
\end{aligned}
$$

where $\underline{\sigma}(\cdot)$ denotes the minimum singular value, and $\bar{e}_{i}\left(k_{l}\right)$ is the mean estimation error of the incorrect filter at time $k_{l}$. We define $\alpha:=\frac{\lambda_{\min }\left(S_{i}^{-1}\right)}{\lambda_{\max }\left(S_{T}^{-1}\right)}$, and $\beta(k):=$ $\frac{2}{\lambda_{\max }\left(S_{T}^{-1}\right)}\left[\ln \left(\frac{\left|S_{i}\right|}{\left|S_{T}\right|}\right)+\ln \left(\frac{\mu_{T}^{-}(k)}{\mu_{i}^{-}(k)}\right)\right]$. Substituting this along with (12) and (13) in Condition 3 of Proposition 1, we obtain the following condition:

$$
\begin{array}{r}
\bar{\sigma}\left(C_{T} A_{T}\right)^{2} \bar{\sigma}\left(F_{T}\right)^{2\left(\delta_{T}-1\right)}\left\|\bar{e}_{T}\left(k_{l}\right)\right\|^{2}<\beta\left(k+\delta_{T}\right) \\
+\alpha \underline{\sigma}\left(L_{i}\right)^{2} \underline{\sigma}\left(\left[\begin{array}{lll}
F_{i} & G_{i}^{x} & G_{i}^{u}
\end{array}\right]\right)^{2\left(\delta_{T}-1\right)}\left\|\bar{e}_{i}\left(k_{l}\right)\right\|^{2}
\end{array}
$$

To find $\delta_{T}$ explicitly, we try a different approach. We can alternatively write the mean residual of the incorrect filter at time $k_{l}+\delta_{T}, \bar{r}_{i}\left(k_{l}+\delta_{T}\right)$ as:

$$
\begin{aligned}
& C_{T} A_{T} F_{i}^{\delta_{T}-1} \bar{e}_{i}\left(k_{l}\right)+H_{i}^{x} \hat{x}_{i}\left(k_{l}+\delta_{T}-1\right) \\
+ & C_{T} A_{T}\left[F_{i}^{\delta_{T}-2} G_{i}^{x} \hat{x}_{i}\left(k_{l}\right)+\cdots+G_{i}^{x} \hat{x}_{i}\left(k_{l}+\delta_{T}-2\right)\right] \\
+ & C_{T} A_{T}\left[F_{i}^{\delta_{T}-2} G_{i}^{u} u\left(k_{l}\right)+\cdots+G_{i}^{u} u\left(k_{l}+\delta_{T}-2\right)\right] \\
+ & H_{i}^{u} u\left(k_{l}+\delta_{T}-1\right)
\end{aligned}
$$

We denote the last four terms of (15) by $b_{i}\left(k_{l}+\delta_{T}-1\right)$ and $J_{i}\left(k_{l}+\delta_{T}\right):=\alpha\left\|b_{i}\left(k_{l}+\delta_{T}-2\right)\right\|^{2}+\beta\left(k_{l}+\delta_{T}\right)$. Using this in Condition 3 of Proposition 1 and combining it with (14), we get:

Proposition 2: The correct mode can be detected $\delta_{T}$ time steps after a mode transition if Condition 1 of Proposition 1 holds and there exists $\delta_{T} \in \mathbb{N}^{+}, \delta_{T}<k_{l+1}-k_{l}, l \in \mathbb{N}^{+}$, $\forall i \neq T$, such that either of the following conditions is true.

$$
\begin{aligned}
& \text { 1) } \bar{\sigma}\left(C_{T} A_{T}\right)^{2} \bar{\sigma}\left(F_{T}\right)^{2\left(\delta_{T}-1\right)}\left\|\bar{e}_{T}\left(k_{l}\right)\right\|^{2}<\beta\left(k+\delta_{T}\right) \\
& +\alpha \underline{\sigma}\left(L_{i}\right)^{2} \underline{\sigma}\left(\left[\begin{array}{ccc}
F_{i} & G_{i}^{x} & G_{i}^{u}
\end{array}\right]\right)^{2\left(\delta_{T}-1\right)}\left\|\bar{e}_{i}\left(k_{l}\right)\right\|^{2} \\
& \text { 2) } \delta_{T}>1+\left\{2 \ln \left[\frac{\sigma\left(F_{i}\right)}{\overline{\bar{\sigma}}\left(F_{T}\right)}\right]\right\}^{-1}\{-\ln \alpha \\
& \left.+2 \ln \left[\frac{\bar{\sigma}\left(C_{T} A_{T}\right)}{\underline{\sigma}\left(C_{T} A_{T}\right)}\right]+2 \ln \left[\frac{\left\|\bar{e}_{T}\left(k_{l}\right)\right\|}{\left\|\bar{e}_{i}\left(k_{l}\right)\right\|}\right]\right\}, \\
& \text { when } J_{i}\left(k_{l}+\delta_{T}\right) \geq 0 \text {. }
\end{aligned}
$$

Note: Although the actual value of

$$
\beta\left(k_{l}+\delta_{T}\right)=\frac{2}{\lambda_{\max }\left(S_{T}^{-1}\right)}\left[\ln \left(\frac{\left|S_{i}\right|}{\left|S_{T}\right|}\right)+\ln \left(\frac{\mu_{T}^{-}\left(k_{l}+\delta_{T}\right)}{\mu_{i}^{-}\left(k_{l}+\delta_{T}\right)}\right)\right]
$$

might be negative, its magnitude is usually not big because it is in a logarithmic scale. Thus, $J_{i}\left(k_{l}+\delta_{T}\right) \geq 0$ is easily satisfied.

In addition, for a two-mode system, if we assume that the estimator converges between transitions and that the mode transition matrix $\Pi$ is diagonally dominant, we obtain the following condition:

Proposition 3: For a hybrid system with two discrete modes, the correct mode can be detected $\delta_{T}$ time steps after a mode transition if there exists $\delta_{T} \in \mathbb{N}^{+}, \delta_{T}<k_{l+1}-k_{l}$, $l \in \mathbb{N}^{+}, i \neq T$, such that

$$
\begin{aligned}
& \alpha \underline{\sigma}\left(L_{i}\right)^{2} \underline{\sigma}\left(\left[\begin{array}{lll}
F_{i} & G_{i}^{x} & G_{i}^{u}
\end{array}\right]\right)^{2\left(\delta_{T}-1\right)}\left\|\bar{e}_{i}\left(k_{l}\right)\right\|^{2} \\
+ & \frac{2}{\lambda_{\max }\left(S_{T}^{-1}\right)}\left[\ln \left(\frac{\left|S_{i}\right|}{\left|S_{T}\right|}\right)-\ln \left(\frac{\pi_{i i}}{1-\pi_{i i}}\right)\right] \\
> & \bar{\sigma}\left(C_{T} A_{T}\right)^{2} \bar{\sigma}\left(F_{T}\right)^{2\left(\delta_{T}-1\right)}\left\|\bar{e}_{T}\left(k_{l}\right)\right\|^{2}
\end{aligned}
$$

Proposition 3 implies that if (16) is satisfied, then the mode probability of the correct mode is definitely greater than those of the other modes after $\delta_{T}$ time steps after a mode transition at time $k_{l}$. Thus, the correct mode is detected $\delta_{T}$ time steps after a mode transition. 


\section{Instantaneous Mode Estimation}

Assuming that the time between discrete transitions is sufficient to allow the Kalman filters and mode probabilities to converge, we follow a procedure similar to Section IV-C, and obtain, for a system with $N$ modes $(N \geq 2)$ :

Proposition 4: The correct mode is detected instantaneously if the following condition holds:

$$
\begin{aligned}
& \alpha \underline{\sigma}\left(L_{i}\right)^{2} \underline{\sigma}\left(\left[\begin{array}{ccc}
F_{i} & G_{i}^{x} & G_{i}^{u}
\end{array}\right]\right)^{2}\left\|\bar{e}_{i}\left(k_{l}-1\right)\right\|^{2} \\
& +\frac{2}{\lambda_{\max }\left(S_{T}^{-1}\right)}\left[\ln \left(\frac{\left|S_{i}\right|}{\left|S_{T}\right|}\right)+\ln \left(\frac{\pi_{j T}}{\pi_{j j}}\right)_{\min }\right] \\
& >\bar{\sigma}\left(C_{T} A_{T}\right)^{2}\left\|\bar{e}_{T}\left(k_{l}-1\right)\right\|^{2}
\end{aligned}
$$

where $\left(\frac{\pi_{j T}}{\pi_{j j}}\right)_{\text {min }}$ is the smallest ratio of off-diagonal to diagonal elements in any row of the $N \times N$ transition matrix.

Some of the expressions in the preceding results may appear intimidating at first sight. They are, however, a source of intuition on the performance of hybrid estimation algorithms, as will be explained in Section V.

\section{E. Exponential convergence of hybrid estimators}

Finally, to present a complete picture, we refer to the authors' previous work [17], in which conditions were derived under which, given a discrete decision time $\delta$, the sojourn time $(\tilde{\Delta})$ is long enough for the error convergence during the period of correct detection $(\tilde{\Delta}-\delta)$ to balance the divergence of the error during the mode mismatch. The discrete decision time in [17] is in fact the mode detection delay that we have derived in the present work. Therefore, combining the two results, we can evaluate the performance of a given hybrid estimator and also find the minimum sojourn time required in each mode to guarantee exponential convergence of the mean square error.

\section{Performance COMPARISONS}

We use the mode estimation delay as a performance metric for comparison of the MMAE and IMM algorithms since a small mode estimation delay usually corresponds to a small estimation error. Analyzing Condition 2 of Proposition 2, we can explain the performance of hybrid estimation algorithms qualitatively. For a small mode estimation delay, the following must be small if $J_{i}\left(k_{l}+\delta_{T}\right) \geq 0$ : $(\forall T \in\{1, \cdots, N\}, \forall i \neq T)$,

$\frac{1}{2} \log \left(\frac{\lambda_{\max }\left(S_{T}^{-1}\right)}{\lambda_{\min }\left(S_{i}^{-1}\right)}\right)+\log \left[\frac{\bar{\sigma}\left(C_{T} A_{T}\right)}{\underline{\sigma}\left(C_{T} A_{T}\right)}\right]+\log \left[\frac{\left\|\bar{e}_{T}\left(k_{l}\right)\right\|}{\left\|\bar{e}_{i}\left(k_{l}\right)\right\|}\right]$

where mode $T$ is the correct mode after the mode transition at time $k_{l}\left(l \in \mathbb{N}^{+}\right)$. Firstly, $\frac{\lambda_{\max }\left(S_{T}^{-1}\right)}{\lambda_{\min }\left(S_{i}^{-1}\right)}$ must be small. Here, the pre-computed residual covariance $S_{i}$ and the steady-state error covariance matrix $P_{i}^{s s}$ computed by Kalman filter $i$ satisfy the algebraic Riccati equation. Therefore, $\frac{\lambda_{\max }\left(S_{T}^{-1}\right)}{\lambda_{\min }\left(S_{i}^{-1}\right)}$ depends only on the system parameters $A_{i}, C_{i}, Q_{i}, R_{i}$ and $A_{T}, C_{T}, Q_{T}, R_{T}$. Thus, by checking the residual covariance matrices for each Kalman filter (which can be done without any measurements), we can tell which mode transition is more detectable than the others. In addition, since $Q_{i}, R_{i}$, $Q_{T}$ and $R_{T}$ are design parameters for the Kalman filters $i$ and $T$, we can make $\frac{\lambda_{\max }\left(S_{T}^{-1}\right)}{\lambda_{\min }\left(S_{i}^{-1}\right)}$ small by adjusting these parameters (Kalman filter tuning) and thus reduce the mode estimation delay. Secondly, if the condition number of $C_{T} A_{T}$ is close to 1 , the second term in (17) becomes small. Thus, we also say which mode is more easily estimated than the others by checking the condition number of $C_{T} A_{T}$ for all $T$. Thirdly, $\frac{\left\|\bar{e}_{T}\left(k_{l}\right)\right\|}{\left\|\bar{e}_{i}\left(k_{l}\right)\right\|}$ must be small, i.e. the mean state estimation errors produced by mode-mismatched Kalman filters should be small (and close to the error produced by the correct Kalman filter).

The mixing step was originally devised to reduce the complexity of the algorithm, yet it also keeps the estimation errors due to filter mismatch small. At the mixing step at each time instant, the IMM shifts the initial conditions for each Kalman filter closer to the (correct) estimate computed by the IMM at the previous time step. Therefore the means of the state estimation errors produced by the incorrect Kalman filters are close to that of the correct Kalman filter. The mode estimation delay of the IMM is therefore smaller than that of the MMAE (which does not have this mixing mechanism), and translates to better performance. Maybeck [1] proposes two ad hoc methods to improve adaptability of the MMAE: enforcing a lower bound on the mode probabilities and adding pseudonoise to the the Kalman filter models. The IMM does both inherently. We now illustrate this through examples.

\section{EXAMPLES}

We first consider mode detection in a simple, onedimensional system such as the one in [12]. The dynamics is of the form $x(k)=a_{i} x(k-1)+b_{i} u(k)+w_{i}(k), y(k)=$ $c_{i} x(k)+v_{i}(k)$, and $u(k)=5 \cos (2 \pi t / 100)$, where the state variables and model parameters are scalar, there are 2 discrete modes, and the input is deterministic and sinusoidal. We estimate the hybrid state sequence from the output sequence using both the MMAE and the IMM. We first check for instantaneous mode detection at a switch using Proposition 5 . We then compute the maximum mode detection delay (or the minimum sojourn time needed to guarantee correct mode detection) using Propositions 3 and 4 . We perform this experiment for various values of the model parameters and compare our predictions with the simulations (Table I-A). As expected, the IMM performs better than the MMAE. Also, since we only compute a conservative estimate of the mode detection delay, it is quite possible that the observed delay is less than the computed bound (as in cases 2 and 3). Fig. 2 shows the mode probabilities and estimates for case 2. The reason for the difference in the performance of the MMAE and IMM algorithms is clear when we consider the estimation errors in Fig. 3. At the mode transition times, the errors of the matched and mismatched filters of the IMM are almost equal, therefore the mode detection delay is small. In our 


\begin{tabular}{|c|c|c|c|c|c|c|c|c|c|c|c|c|c|c|c|c|}
\hline \multicolumn{17}{|c|}{ A. Two-Mode Example: MMAE and IMM Performance vs. Parameters (Monte Carlo Simulation, 100 trials) } \\
\hline \multirow{3}{*}{ Case } & \multirow{3}{*}{ Algorithm } & \multirow{2}{*}{\multicolumn{4}{|c|}{$\begin{array}{l}\text { Mode Parameters } \\
\qquad\left(b_{1}=b_{2}=1\right)\end{array}$}} & \multirow{2}{*}{\multicolumn{4}{|c|}{$\begin{array}{c}\text { Instant Detection } \\
\text { Condition }\end{array}$}} & \multicolumn{5}{|c|}{ Mode detection delay } & \multirow{2}{*}{\multicolumn{2}{|c|}{$\frac{\lambda_{\max }\left(S_{T}^{-1}\right)}{\lambda_{\min }\left(S_{i}^{-1}\right)}$}} \\
\hline & & & & & & & & & & \multicolumn{3}{|c|}{ Predicted } & \multicolumn{2}{|c|}{ Observed } & & \\
\hline & & a1 & a2 & $\mathrm{c} 1$ & c2 & & & $1-$ & & $\delta_{1}^{*}$ & & $\delta_{2}^{*}$ & $\delta_{1}$ & $\delta_{2}$ & $\mathrm{~T}=1$ & $\mathrm{~T}=2$ \\
\hline \multirow[t]{2}{*}{1} & \multirow{2}{*}{$\begin{array}{l}\text { MMAE } \\
\text { IMM }\end{array}$} & \multirow[t]{2}{*}{0.95} & \multirow[t]{2}{*}{0.25} & \multirow[t]{2}{*}{1} & \multirow[t]{2}{*}{0.80} & \multicolumn{2}{|l|}{$\sqrt{ }$} & \multicolumn{2}{|l|}{$x$} & 0 & \multicolumn{2}{|c|}{2} & 0 & 2 & \multirow[t]{2}{*}{0.48} & \multirow[t]{2}{*}{2.09} \\
\hline & & & & & & $\sqrt{ }$ & & $\times$ & & 0 & & 2 & 0 & 2 & & \\
\hline \multirow[t]{2}{*}{2} & \multirow{2}{*}{$\begin{array}{c}\text { MMAE } \\
\text { IMM }\end{array}$} & \multirow[t]{2}{*}{0.85} & \multirow[t]{2}{*}{0.85} & \multirow[t]{2}{*}{0.80} & \multirow[t]{2}{*}{0.2} & $x$ & & \multicolumn{2}{|l|}{$x$} & 6 & \multicolumn{2}{|c|}{2} & 4 & 0 & \multirow[t]{2}{*}{1.29} & \multirow[t]{2}{*}{0.77} \\
\hline & & & & & & $x$ & & $\sqrt{ }$ & & 2 & & 0 & 0 & 0 & & \\
\hline \multirow[t]{2}{*}{3} & \multirow{2}{*}{$\begin{array}{c}\text { MMAE } \\
\text { IMM }\end{array}$} & \multirow[t]{2}{*}{0.95} & 0.85 & 1.0 & 0.40 & $x$ & & $x$ & & 10 & & 2 & 5 & 1 & 1.13 & 0.89 \\
\hline & & & & & & $\sqrt{ }$ & & $\sqrt{ }$ & & 0 & & 0 & 0 & 0 & & \\
\hline & B. $\mathrm{Tl}$ & ee-M & de Exa & nple: & IMAE & nd 1 & MM P & forn & nce & $\mathrm{Pa}$ & ime & ers (N & nte & arlo Sim & ion, 100 & \\
\hline Case & Algorithm & & & lode $\mathrm{P}$ & meters & & & & & det & tion & delay & & & $\operatorname{tax} \frac{\lambda}{\lambda}$ & \\
\hline & & & & $=b_{2}$ & $b 3=$ & & & & edic & & & Observ & & & & \\
\hline & & a1 & a2 & a3 & $\mathrm{c} 1$ & $\mathrm{c} 2$ & c3 & $\delta_{1}^{*}$ & $\delta_{2}^{*}$ & $\delta_{3}^{*}$ & $\delta_{1}$ & $\delta_{2}$ & $\delta_{3}$ & $\mathrm{~T}=1$ & $\mathrm{~T}=2$ & $\mathrm{~T}=3$ \\
\hline 1 & MMAE & 1.2 & 0.25 & 0.95 & 0.80 & 1.0 & 0.80 & 2 & 0 & 7 & 1 & 0 & 5 & 1.2 & 0.80 & 1.26 \\
\hline & IMM & & & & & & & 1 & 0 & 5 & 1 & 0 & 4 & & & \\
\hline
\end{tabular}

TABLE I

(A) Two MOdE AND (B) THREe MOdE EXAMPLES

example, (17) simply reduces to $\frac{\lambda_{\max }\left(S_{T}^{-1}\right)}{\lambda_{\min }\left(S_{i}^{-1}\right)}$ in the case of the IMM, and Table I-A shows that as predicted, the smaller the value of (17), the smaller the mode detection delay. The biggest advantage of this result is that given a system and its error bounds, this gives us a way to determine a priori transitions to which modes are the most detectable. Results for a three-mode example are shown in Table I-B.

We now consider an aircraft tracking example, with two discrete modes, the constant velocity (CV) mode and the coordinated turn (CT) mode. The dynamics of both modes is given in the Appendix. The mode changes occur at time $=45$ seconds (CV to CT) and at time $=56$ seconds ( CT to CV). Using Proposition 2 for the IMM, we find that the mode estimation delay for the mode switching from $\mathrm{CV}$ to $\mathrm{CT}$ is $\delta_{c t}=1$, and from CT to $\mathrm{CV}$ is $\delta_{c v}=2$. We therefore expect the mode switching from mode $\mathrm{CV}$ to $\mathrm{CT}$ to be more detectable than the mode switching from mode CT to $\mathrm{CV}$. Similarly, we obtain $\delta_{c t}=7$ and $\delta_{c v}=12$ for the MMAE. Fig. 4 shows that the simulations validate these predictions well. The IMM performs better than the MMAE; and the mode estimation delays for both the IMM (1 for CV to CT; 2 for CT to CV) and the MMAE (6 for CV to CT; 10 for CT to $\mathrm{CV}$ ) are close to those predicted, and within the bounds.

\section{CONCLUSIONS}

Although several hybrid estimation algorithms have existed for many years, the issues of their performance and limitations have not been addressed in much detail. In this paper, we have performed a detailed steady-state and transient analysis of these algorithms and derived necessary conditions for correct mode detection, bounds on their performance in terms of the mode detection delay and the minimum sojourn time, and also proposed a way to predict a priori which mode transitions are the easiest to detect, and validated our results using simulated experiments. Most importantly, our results give a mathematical yet intuitive explanation for why the IMM algorithm achieves its high levels of performance in the estimation of stochastic linear hybrid systems.

\section{ACKNOWLEDGEMENTS}

This research is supported by ONR under MURI contract N00014-02-1-0720 and by an NSF Career Award (ECS-9985072). H. Balakrishnan is supported by a Stanford Graduate Fellowship.

\section{REFERENCES}

[1] P.S. Maybeck. Stochastic Models, Estimation, and Control, volume 2. New York: Academic Press, 1982.

[2] Y. Bar-Shalom, X.R. Li, and T. Kirubarajan. Estimation with Applications to Tracking and Navigation. John Wiley \& Sons, 2001.

[3] D.D. Sworder and J.E. Boyd. Estimation problems in Hybrid Systems. Cambridge University Press, 1999.

[4] R.M. Hawkes and J.B. Moore. Performance bounds for adaptive estimation. Proceedings of the IEEE, 64(8):1143-1150, August 1976.

[5] D.T. Magill. Optimal adaptive estimation of sampled stochastic processes. IEEE Transactions on Automatic Control, 10(4):434-439, October 1965.

[6] D.G. Lainiotis and F.L. Sims. Performance measures for adaptive Kalman estimators. IEEE Transactions on Automatic Control, 15(2):249-250, April 1970.

[7] F.L. Sims, D.G. Lainiotis, and D.T. Magill. Recursive algorithm for the calculation of the adaptive Kalman filter weighting coefficients. IEEE Transactions on Automatic Control, 14(2):215-218, April 1969.

[8] D.G. Lainiotis. Optimal adaptive estimation: Structure and parameter adaptation. IEEE Transactions on Automatic Control, 16(2):160-170, April 1971.

[9] Y. Baram and N.R. Sandell. Consistent estimation on finite parameter sets with application to linear system identification. IEEE Transactions on Automatic Control, 23(3):451-454, June 1978.

[10] Y. Baram. A sufficient condition for consistent discrimination between stationary Gaussian models. IEEE Transactions on Automatic Control, 23(5):958-960, October 1978.

[11] M.J. Caputi. A necessary condition for effective performace of the multiple model adaptive estimator. IEEE Transactions on Aerospace and Electronic Systems, 31(3):1132-1138, July 1995. 

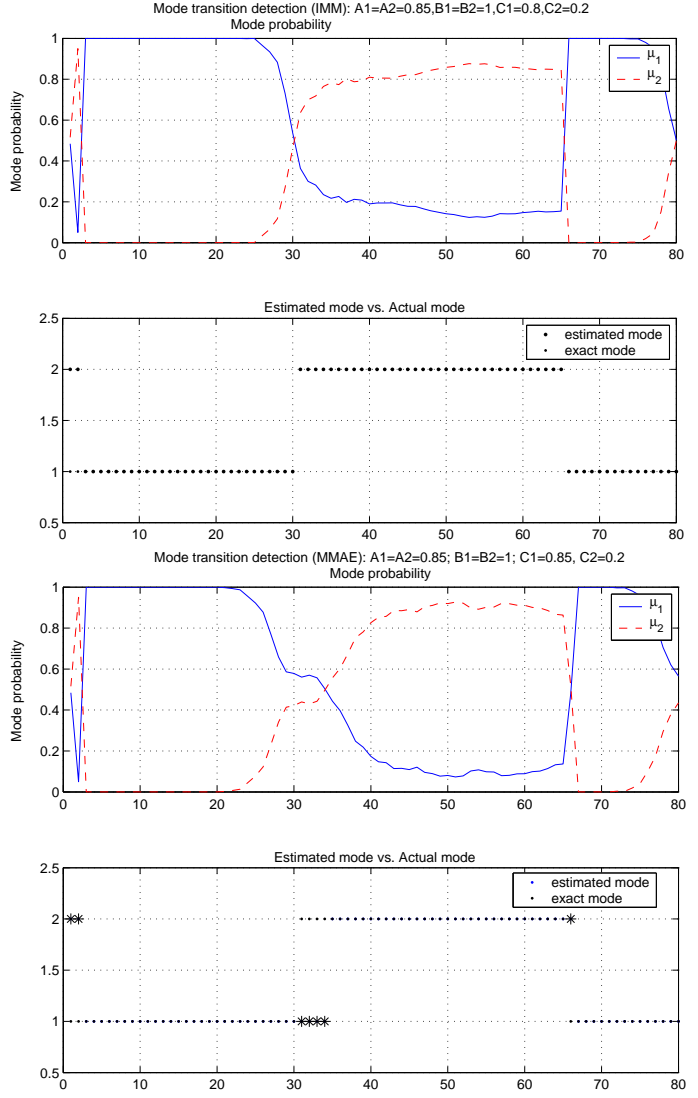

Fig. 2. Mode estimates by (top) IMM and (bottom) MMAE: 100 trial MC simulation results. The asterisks denote mode estimation delay.

[12] H.A.P. Blom and Y. Bar-Shalom. The interacting multiple model algorithm for systems with Markvian switching coefficients. IEEE Transactions on Automatic Control, 33(8):780-783, 1988.

[13] X.R. Li and Y. Bar-Shalom. Design of an interacting multiple model algorithm for air traffic control tracking. IEEE Transactions on Control Systems Technology, 1(3):186-194, September 1993.

[14] P.D. Hanlon and P.S. Maybeck. Characterization of Kalman filter residuals in the presence of mismodeling. In Proceedings of the $37^{\text {th }}$ IEEE Conference on Decision and Control, pages 1254-1259, Tampa, FL, December 1998.

[15] R.A. Horn. Matrix Analysis. Cambridge University Press, 1985.

[16] T. Kailath. Linear Systems. Prentice Hall, 1980.

[17] I. Hwang, H. Balakrishnan, and C. Tomlin. Observability criteria and estimator design for stochastic linear hybrid systems. In Proceedings of IEE European Control Conference, Cambridge, UK, September 2003.

\section{APPENDIX}

\section{AIRCRAFT KINEMATIC MODEL:}

$$
\begin{aligned}
x(k+1)= & {\left[\begin{array}{cccc}
1 & T & 0 & 0 \\
0 & 1 & 0 & 0 \\
0 & 0 & 1 & T \\
0 & 0 & 0 & 1
\end{array}\right] x(k)+\left[\begin{array}{cc}
\frac{T^{2}}{2} & 0 \\
T & 0 \\
0 & \frac{T^{2}}{2} \\
0 & T
\end{array}\right] u_{i}(k) } \\
& +w_{i}(k) \\
y(k)= & {\left[\begin{array}{llll}
1 & 0 & 0 & 0 \\
0 & 0 & 1 & 0
\end{array}\right] x(k)+v_{i}(k),(i \in\{C V, C T\}) }
\end{aligned}
$$

where $x=\left[\begin{array}{llll}x_{1} & \dot{x}_{1} & x_{2} & \dot{x}_{2}\end{array}\right]^{T}$ where $x_{1}$ and $x_{2}$ are the position coordinates, $u=\left[\begin{array}{ll}u_{1} & u_{2}\end{array}\right]^{T}$ where $u_{1}$ and $u_{2}$ are the acceleration components. The control input is given by: $u_{C V}=\left[\begin{array}{ll}0 & 0\end{array}\right]^{T}$ in CV mode, $u_{C T}=\left[\begin{array}{ll}1.5 & 1.5\end{array}\right]^{T}$ in CT mode, $T$ is the sampling interval, $w_{i}$ is the process noise, and $v_{i}$ is the sensor noise. We choose an operating velocity of 150 knots.
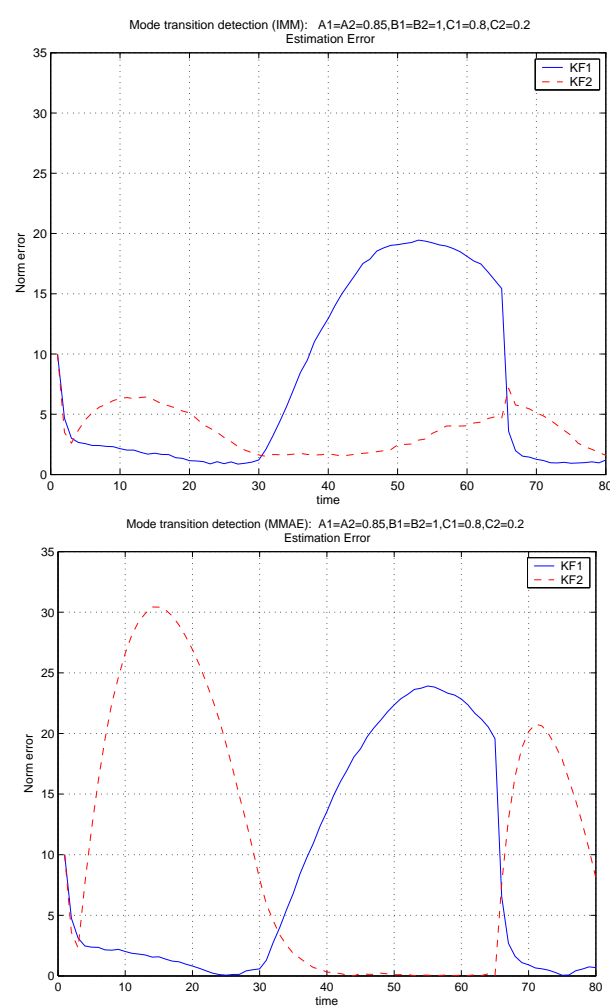

Fig. 3. Estimation error by (top) IMM and (bottom) MMAE: 100 trial MC simulation results
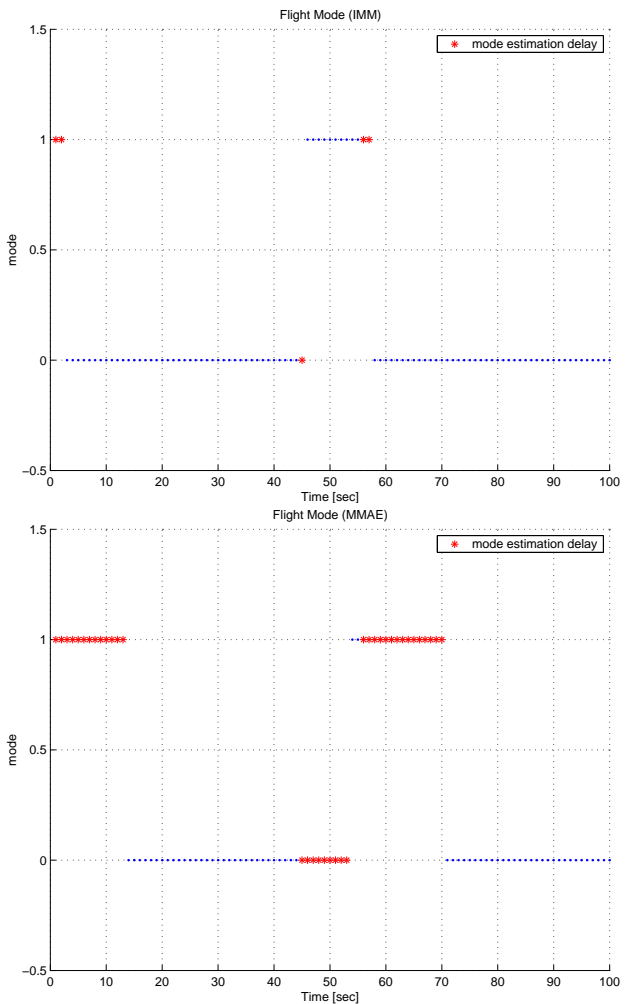

Fig. 4. Aircraft mode estimates by (top) IMM and (bottom) MMAE: 100 trial MC simulation results (mode $\mathrm{CV}=0$, mode $\mathrm{CT}=1$ ). 